\title{
Quantitative Evaluation of a Telerobotic System for Vascular Ultrasound Measurement on a Short Arm Human Centrifuge
}

\author{
Timo Frett ${ }^{1}$ (D) $\cdot$ Guido Petrat ${ }^{1} \cdot$ Michael Arz $^{1} \cdot$ Carole Leguy ${ }^{1,2}$ \\ Received: 1 September 2020 / Accepted: 1 December 2020 / Published online: 23 January 2021 \\ (C) The Author(s) 2021
}

\begin{abstract}
Artificial Gravity generated by Short Arm Human Centrifuges is a promising multi-system countermeasure for physiological deconditioning during long duration space flights. To allow a continuous assessment of cardiovascular hemodynamics during centrifugation, a telerobotic robotic system holding an ultrasound probe has been installed on a Short Arm Human Centrifuge. A feasibility study was conducted to define the use capabilities and limitations of such a novel method. The objective of this study is to estimate the reproducibility and precision of remotely controlled vascular ultrasound assessment under centrifugation by assessing peripheral vascular diameter and wall distension. Four repeated centrifugation runs of $5 \mathrm{~min}$, with $2.4 \mathrm{~g}$ at feet level, were performed including a $15 \mathrm{~min}$ rest between each run for a group of eight healthy male volunteers. Vascular diameter and distention were assessed for the common carotid artery (CCA) and the femoral artery (FA) by ultrasound imaging using a $10 \mathrm{MHz}$ linear array probe (Mylab1, Esaote). Ultrasound measurements were consecutively performed: a) by an expert user in hand-held mode in standing as well as supine position, b) using the telerobotic arm without centrifugation as baseline and c) using the telerobotic arm during centrifugation. Vascular responses were compared between baseline and under centrifugation. Inter-, intra-registration and group variability have been assessed for hand-held and remotely controlled examination. The results show that intra-registration variability, $\sigma_{\mathrm{h}}$, was always smaller than inter-registration variability, $\sigma_{\mathrm{m}}$, that is in turned smaller than the inter-subject variability $\sigma_{\mathrm{g}}\left(\sigma_{\mathrm{h}}<\sigma_{\mathrm{m}}<\sigma_{\mathrm{g}}\right)$. Centrifugation caused no significant changes in CCA diameter but a lower carotid distension compared to manual and robotic ultrasound in supine position $(p<0.05)$. Femoral diameter was significantly decreased in hypergravity compared to robotic sonography without centrifugation. A good reproducibility and precision of the remotely controlled vascular ultrasound assessment under centrifugation could be demonstrated. In conclusion, arterial wall dynamics can be precisely assessed for the CCA and femoral artery during centrifugation using a telerobotic ultrasound measurement system. Potential improvements to further enhance reproducibility and safety of the system are discussed.
\end{abstract}

Keywords Telerobotic $\cdot$ Artificial gravity $\cdot$ Ultrasound imaging $\cdot$ Vascular response $\cdot$ Space physiology

\section{Introduction}

In preparation for the next phase of manned long term spaceflight exploration a more profound understanding of cardiovascular responses to gravitational load is crucial. While standing upright on Earth, a head-to-foot gravitational force results in regular fluid

Timo Frett

Timo.frett@dlr.de

1 German Aerospace Center (DLR), Institute of Aerospace Medicine, 51147 Cologne, Germany

2 Institute of Measuring and Sensor Technology, Ruhr West University of Applied Science, Mülheim an der Ruhr, Germany shifts to and from the head. In microgravity this process alters and elicits a decrease in cardiac output that can induce postspaceflight orthostatic intolerance which remains a major issue for astronauts (Lee et al. 2015; Gunga et al. 2016).

Artificial Gravity generated by short arm centrifugation is considered to counteract space flight-induced cardiovascular deconditioning as it induces a hydrostatic pressure column along the body long axis to simulate Earth's gravity (Stenger et al. 2012; Clément and Pavy-Le Traon 2004; Iwase et al. 2007; Linnarsson et al. 2015). Recent design models recommend intermittent short-radius centrifugation (radius $<10 \mathrm{~m}$ ) for exercise activities during long term missions that will result in a significant gravity gradient along the body longitudinal axis (Clement 2017; Simón et al. n.d.). A better knowledge on cardiovascular responses to a steep gravity gradient is 
essential to develop future countermeasures based on acritical gravity.

Using functional ultrasound imaging, it is possible to assess non-invasively and in real time, arterial diameter and wall distension at peripheral arteries as the Common Carotid Artery (CCA) or the femoral artery (FA). These methods, based on radio-frequency signal techniques, are recognized as the gold standard in arterial wall dynamics measurement. The automatic detection of the artery wall directly from radiofrequency signals has the advantage of being more reproducible and user-independent than manual detection by the end user (Leguy et al. 2009; Meinders and Hoeks 2004; Brands et al. 1999). An accurate and stable probe positioning is crucial for such measurements and must be continuously adjusted by the sonographer. During centrifugation, the sonographer must remain remote from the subject in the control room. Therefore, only a telerobotic system can allow such measurements. While telerobotic systems have been developed for various clinical applications (Dogangil et al. 2010; Marescaux and Rubino 2003; Evans et al. 2018) their employment on a human centrifuge requires specific safety measures. For this purpose, a telerobotic ultrasound system for vascular sonography has been built on the DLR Short Arm Human Centrifuge (Frett et al. 2014a) that can be used in hypergravity conditions up to $+6 \mathrm{~g}$. The objective of the study is to estimate the reproducibility and precision of a remotely controlled realtime assessment of arterial diameter and wall distension during centrifugation using functional telerobotic ultrasound imaging. To our knowledge, haemodynamic measurements using ultrasound functional imaging during centrifugation were never been performed on humans. We compared measurements of carotid and femoral diameter during centrifugation using the telerobotic ultrasound system with hand-held and telerobotic measurements before centrifugation. Results were analyzed for inter-subject in terms of inter-registration variability as well as intra-measurement variability.

\section{Methods}

Study Subjects In accordance with the ethical committee North Rhine, Germany (Communications Reference Number 2014258) 9 healthy and non-smoking male subjects were recruited. Their average age was $30 \pm 5$ years, their average weight $80 \pm 9.2 \mathrm{~kg}$, and their average height $182 \pm 4 \mathrm{~cm}$. All subjects passed standard medical screening for the centrifuge experiments and having provided written informed consent.

Telerobotic Ultrasound Ultrasound measurements were recorded while subjects lying supine on DLR's Short Arm Human Centrifuge (SAHC) (Frett et al. 2016; Frett et al. 2014a; Frett et al. 2014b). A point-of-care ultrasound system
(ESAOTE MyLabOne, Italy) equipped with a $10 \mathrm{Mhz}$ vascular probe was customized by the manufacturer to be remotely controllable from the centrifuge control room and fixed safely on the centrifuge.

The ultrasound scanner settings are remotely controllable from the sonographer and a 1:1 reproduction of the normal scanner display was carried to a monitor in the control room. The ultrasound probe is mounted on the tip of the telerobotic arm that was first positioned above the subject (see Figs. 1 and 2). By using a control panel in the centrifuge control room, the sonographer can adjust the probe position in all 6 degrees of freedom within safety limits also while the centrifuge is spinning. The robotic arm has a position accuracy of $\pm 1 \mathrm{~mm}$. Pressure on the subject's skin is controlled by a pressure system that limit the contact pressure from 1 to a maximum of $100 \mathrm{~N}$. This pressure was carefully adjusted depending on the probe position and overall limited to $20 \mathrm{~N}$ (Frett et al. 2014a). During centrifugation, the current probe position was monitored by two video cameras on different angles.

On the centrifuge, subjects were secured wearing a 6-point safety belt (Schroth, Germany) and non- invasively monitored by an attending physician (electrocardiogram, manual blood pressure and oxygen saturation). Subjects were told not to move during the measurement and were blindfolded to reduce the risk of motion sickness symptoms.

Protocol All subjects participated in one experiment session on the Short-Arm Human Centrifuge at the DLR Institute of Aerospace Medicine, Cologne (Germany). An anamnesis and familiarization with the centrifuge' robotic arm were performed prior to the experiment. Baseline measurements included height, body weight, distance head (apex) to CCA (around $2 \mathrm{~cm}$ below chin) were assessed.

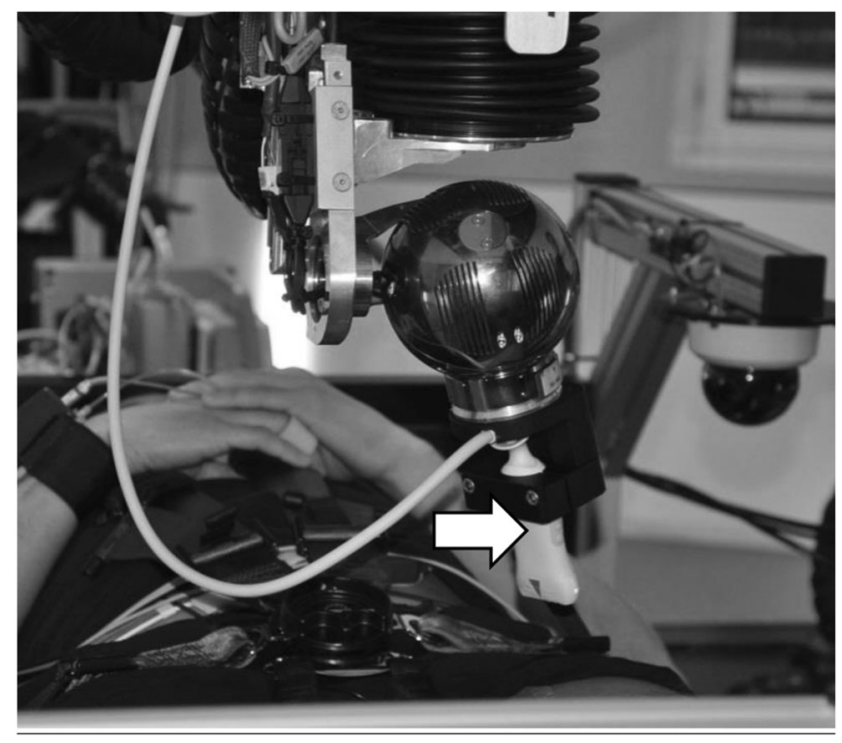

Fig. 1 Remotely controlled robotic system with vascular probe mounted at the tip (arrow) 
Fig. 2 Remote control panel for the robotic ultrasound system. a Safety button b Slide control to adjust probe pressure on subject's skin. Maximal applicable pressure with the slide control can be limited in the software between 1 and $100 \mathrm{~N}) \mathbf{c} 2$-dimensional linear axes joystick $\mathbf{d} 3$ dimensional rotatory control

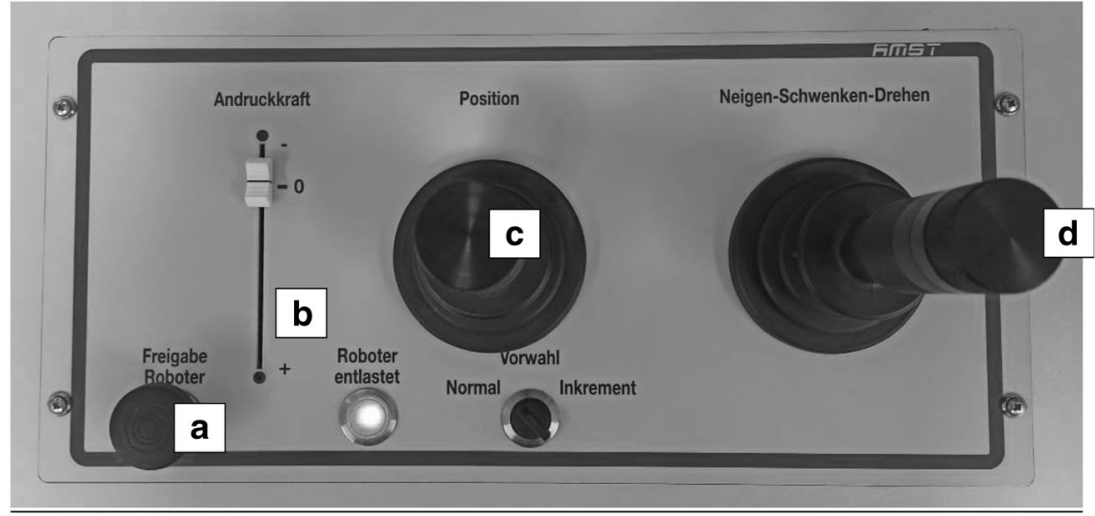

Before centrifugation optimal probe position for sonography of CCA and FA were marked on the subject's skin with medical tape. Baseline measurements of the carotid and femoral mean diameter $D$ and vessel wall distension, $\Delta D$ (difference between systolic and diastolic diameter), were recorded in randomized order (Fig. 3):

- by hand in supine position, condition: 'hand-supine',

- with the ultrasound probe attached at the robotic arm from supine position without centrifugation, condition: 'robotic-static', and,

- with the remotely controlled robotic arm during centrifugation at $+2.4 \mathrm{Gz}$ at feet: condition: 'roboticcentrifugation'.

Centrifuge speed and subject axis position was calculated for a g-load of $+2.4 \mathrm{Gz}$ at feet level and $+1.0 \mathrm{Gz}$ at the heart level. Resulting $+G z$-load at heart and carotid level were recorded depending on subject's body height and measured distances CCA-Apex and CCA-heart. The centrifuge profile consisted of four hyper-G phases with constant individual RPM (rotation per minute). Mean spin rate of the centrifuge was therefore $29 \pm 0.4 \mathrm{rpm}$ with $+0.9 \mathrm{Gz}$ at Carotis, $+1.0 \mathrm{Gz}$ at heart and $+2.4 \mathrm{Gz}$ at feet. To ensure subjects orthostatic stability at the combined high g-load and steep gradient, each centrifuge run was limited to $5 \mathrm{~min}$. Assessment of arterial diameter and wall distention from carotid and femoral artery were randomized between the subjects Fig. 4 .

\section{Statistical Analysis}

The variability of the assessed vessel diameter and wall distension was investigated under the different conditions. We differentiated, for each condition, among the

- intra-registration variability, $\sigma_{\mathrm{h}}$, between the consecutive heartbeats within single measurements, (Eq. 1),

- inter-registration variability $\sigma_{\mathrm{m}}$ between repeated measurements, (Eq. 2), and,

- inter-subject variability $\sigma_{\mathrm{g}}$ within the volunteer of the group (Eq. 3).

The intra-registration variability $\sigma_{\mathrm{h}}$ is defined as:

$\sigma_{h}=\sqrt{\frac{\sum_{v} \sum_{m} \sum_{b}\left(X_{v, m, b}-\bar{X}_{v, m}\right)^{2}}{\sum_{v} \sum_{m}\left(b_{v, m}\right)-n_{m}}}$

with $X_{v, m, b}$ a parameter representing either the assessed average diameter $(D)$ or the vessel wall distension $(\Delta D)$ for the volunteer $v$, in measurement $m$ at heart-beat $b$. The average parameter for the measurement $m$ of volunteer $v$ is given by
Fig. 3 Measurement during all four study conditions: manual sonography standing and in supine position, robotic sonography with and without centrifugation in supine position
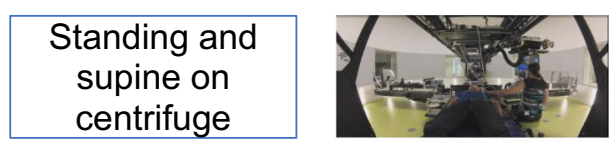

\begin{tabular}{l} 
Supine on \\
centrifuge \\
\hline
\end{tabular}

Supine on centrifuge + 2.4 Gz-Load
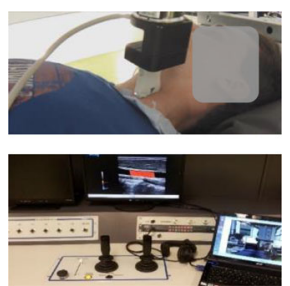

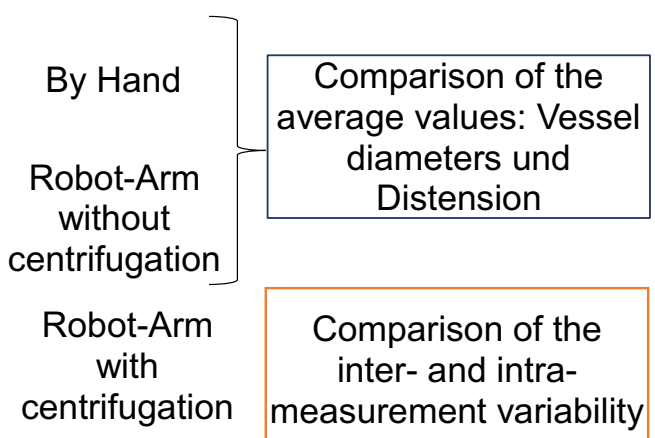




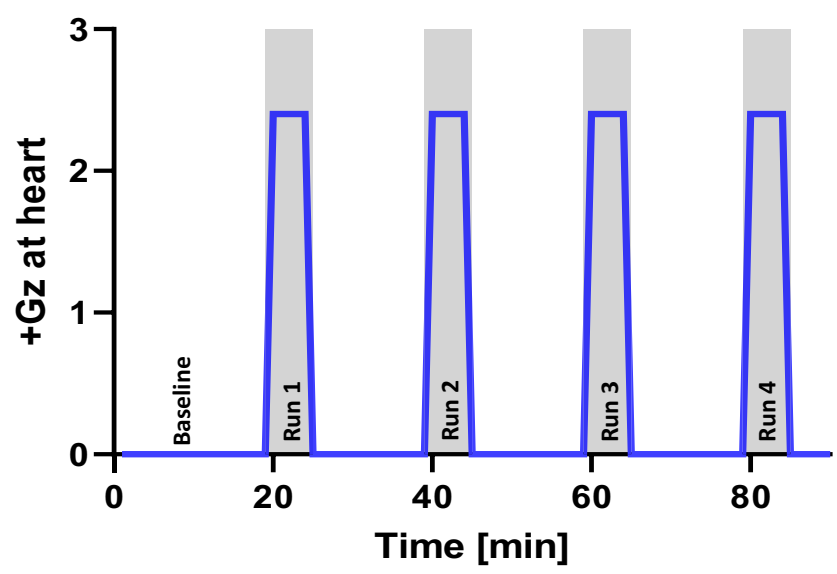

Fig. 4 Centrifuge profile with four sessions of artificial gravity at $+2.7 \mathrm{Gz}$ @ heart level

$\bar{X}_{v, m}$. The number of beats for a measurement $m$ for the volunteer $v$ is given by $b_{v, m}$ and the total number of measurements by $n_{m}$.

The inter-measurement variability $\sigma_{m}$ is defined as:

$\sigma_{m}=\sqrt{\frac{\sum_{v} \sum_{m}\left(X_{v, m}-\bar{X}_{v}\right)^{2}}{\sum_{v}\left(m_{v}\right)-v}}$

with $X_{v, m}$ the parameter value for the volunteer $v$ and measurement $m, \bar{X}_{v}$ the average parameter for the volunteer $v$. The number of measurements for a volunteer $v$ is given by $m_{v}$, and, the number of volunteers by $v$.

The group-variability (or inter-subject variability) $\sigma_{g}$ is given by:

$\sigma_{g}=\sqrt{\frac{\sum_{v}\left(X_{v}-\bar{X}\right)^{2}}{v-1}}$

with $X_{v}$ the parameter average for the volunteer $v$ and $\bar{X}$ the group average.

Results for each condition were analyzed using paired ttests to compare each condition. Mean values were reported with standard deviation. All statistical tests were conducted using IBM SPSS version 21 (IBM Corp., USA) with $\alpha<$ 0.05 indicating significance.

\section{Results}

Two subjects were excluded, one due to pre-syncopal symptoms during centrifugation, and one other because of technical problems. From the remaining seven subjects, complete datasets (with valid ultrasound hemodynamic measurements during centrifugation) have been obtained from six subjects for the carotid artery with a success rate of $71 \%$, and, four for the femoral artery with a success rate of $57 \%$.

Mean values for heart rate, systolic and diastolic blood pressure are $74 \pm 20.17 \mathrm{bpm}, 119.4 \pm 9.27 \mathrm{mmHg}$ and 69.4 $\pm 9.0 \mathrm{mmHg}$ before centrifugation (rest supine), and, $68 \pm$ $18.0 \mathrm{bpm}, 122.6 \pm 5.9 \mathrm{mmHg}$ and $69.1 \pm 7.5 \mathrm{mmHg}$ after centrifugation (recovery), with no significant differences. During centrifugation, mean values are $95 \pm 15 \mathrm{bpm}$ for heart rate, $126.4 \pm 5 \mathrm{mmHg}$ for systolic and $83.8 \pm 8.6 \mathrm{mmHg}$ for diastolic blood pressure (Figs. 5, 6 and 7) with significant increase of heart rate, systolic and diastolic blood pressure $(p<0.05)$. No significant differences for heart rate and blood pressure are obtained between the four centrifuge runs.

The measured CCA average diameter $\left(D_{C C A}\right)$ for manual ultrasound in supine position are $7.1 \pm 0.2 \mathrm{~mm}$, the vessel wall distension $\left(\Delta D_{C C A}\right) 565 \pm 134 \mu \mathrm{m}$. For robotic sonography without centrifugation $D_{C C A}$ are $6.9 \pm 0.3 \mathrm{~mm}$ and $\triangle D_{C C A}$ $493 \pm 120 \mu \mathrm{m}$ and $6.7 \pm 0.6 \mathrm{~mm}$ and $334 \pm 87 \mu \mathrm{m}$ for robotic sonography during centrifugation (Figs. 8 and 9). Centrifugation induces no significant difference in carotid diameter for all three conditions. However, the carotid distension is significantly lower during centrifugation compared to manual, and, robotic $(p<0.05)$ sonography in supine position without centrifugation $(p<0.05)$.

For femoral artery average measured diameter $\left(D_{F A}\right)$ and distension $\left(\Delta D_{F A}\right)$ are $7,2 \pm 0.6 \mathrm{~mm}$ and $168 \pm 22 \mu \mathrm{m}$ for manual ultrasound in supine position, $7.2 \pm 0.4 \mathrm{~mm}$ and 158 $\pm 27 \mu \mathrm{m}$ for robotic sonography without centrifugation, and,
Fig. 5 Average values for heart rate, systolic and diastolic blood pressure with standard deviation during centrifugation. Baseline measurements were taken before centrifugation (pre) and during the experiment including breaks between centrifuge runs until end of protocol (post)

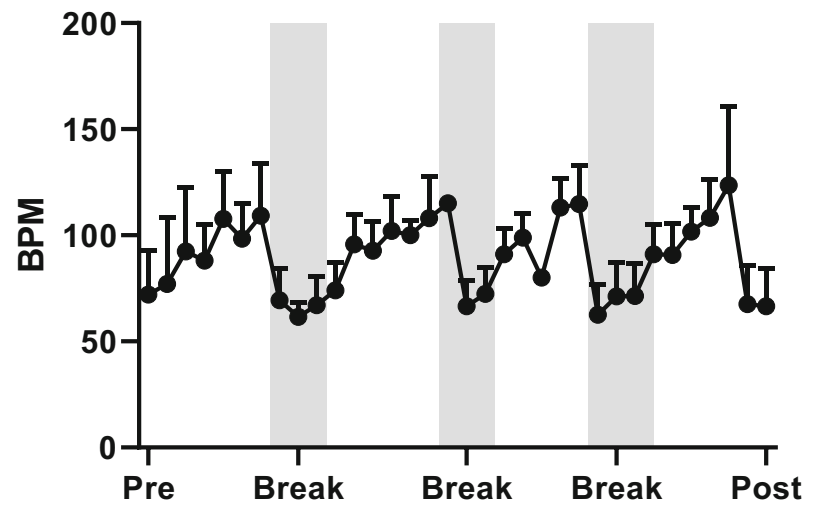


Fig. 6 Average values for heart rate, systolic and diastolic blood pressure with standard deviation during centrifugation. Baseline measurements were taken before centrifugation (pre) and during the experiment including breaks between centrifuge runs until end of protocol (post)

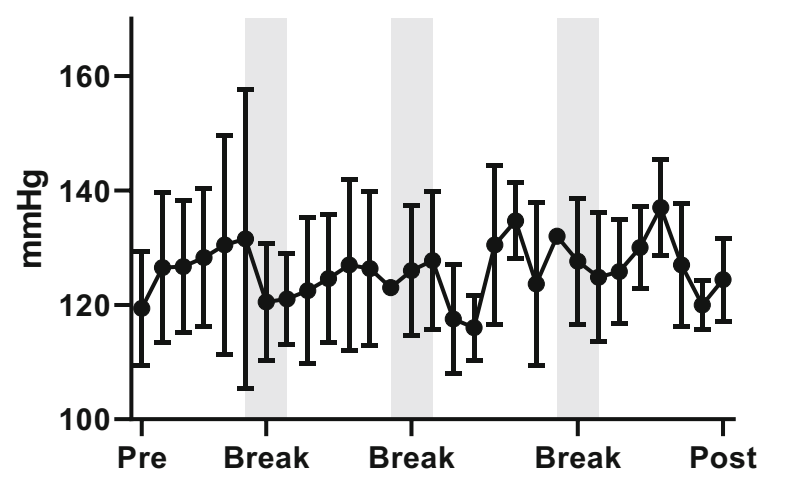

- Systolic blood pressure
$6.3 \pm 0.6 \mathrm{~mm}$ and $159 \pm 67 \mu \mathrm{m}$ for robotic sonography during centrifugation (Figs. 10 and 11). The decrease in diameter during centrifugation is significant compared with robotic sonography, however it does not reach significance with manual ultrasound in supine without centrifugation $(p=0.1)$. No significant difference in distension could be measured in any condition Figs. 12 and 13.

For both the $D_{C C A}$, and for $D_{F A}$, the intra-registration variability, $\sigma_{\mathrm{h}}$, is smaller than the inter-registration variability $\sigma_{\mathrm{m}}$, that is in turned smaller than the inter-subject variability $\sigma_{\mathrm{g}}$ ( $\sigma_{\mathrm{h}}<\sigma_{\mathrm{m}}<\sigma_{\mathrm{g}}$ ) for the 3 conditions: hand-held, remotely controlled without and with centrifugation (Tables 1 and 2). Furthermore, the results show that for both the $D_{C C A}$ and the $D_{F A}$ the intra-measurement variability are not increased between hand-held and remotely controlled measurements without and with centrifugation (Tables 1 and 2).

For the carotid distensibility, $\triangle D_{C C A}$, the intra-registration variability, $\sigma_{\mathrm{h}}$, is smaller than the inter-registration variability $\sigma_{\mathrm{m}}$, that is in turned smaller or equal to the inter-subject variability $\sigma_{\mathrm{g}}\left(\sigma_{\mathrm{h}}<\sigma_{\mathrm{m}} \leq \sigma_{\mathrm{g}}\right)$ for the 3 conditions: hand-held, remotely controlled without and with centrifugation (Table 3 ). The distensibility measurement at the femoral, $\Delta D_{F A}$, do not show a consistent order among the three measures of variability (Table 4).

\section{Discussion}

In this study, a quantitative evaluation of a telerobotic system for vascular ultrasound measurement on a short arm human centrifuge was investigated. To the author's knowledge, hemodynamic measurements using remotely controlled ultrasound functional imaging during centrifugation were never been performed on humans. Repeated assessment of the vessel means diameter and wall distension of carotid and femoral artery showed a good feasibility under static condition, as well as under, hypergravity conditions (with $+2.4 \mathrm{Gz}$ at feet level) on a Short Arm Human Centrifuge. No significantly differences were obtained between the reference hand-held measurements and the one obtained using the robotic-arm in static conditions. The success rate at the carotid and femoral arteries were $71 \%$ and $43 \%$ during centrifugation. Specificity of the measurements during centrifugation could be demonstrated by the intra-measurements variability that are lower than the inter-measurement variability.

The comparison of heart rate and blood pressure values during supine baseline and centrifugation showed a significant increase due to g-load. During centrifugation increased femoral diameter were expected as a result of fluid shifts toward lower extremities. Recent findings (Verma et al. 2018; Goswami et al. 2015) indicating that centrifugation with +2 $\mathrm{Gz}$ at feet result in analogue responses of cardiovascular system and autonomic blood pressure control to standing in Earth's gravity. The ultrasound measurements considered in this study correspond to values find in literature. In Leguy et al. 2009 (3), an average femoral diameter (at rest in supine) on a similar subject group (6 healthy young male) of $4.1 \pm$ $0.5 \mathrm{~mm}$ is reported, while in this study this parameter equals $6.6 \pm 1.0 \mathrm{~mm}$. The higher average diameter and group
Fig. 7 Average values for heart rate, systolic and diastolic blood pressure with standard deviation during centrifugation. Baseline measurements were taken before centrifugation (pre) and during the experiment including breaks between centrifuge runs until end of protocol (post)

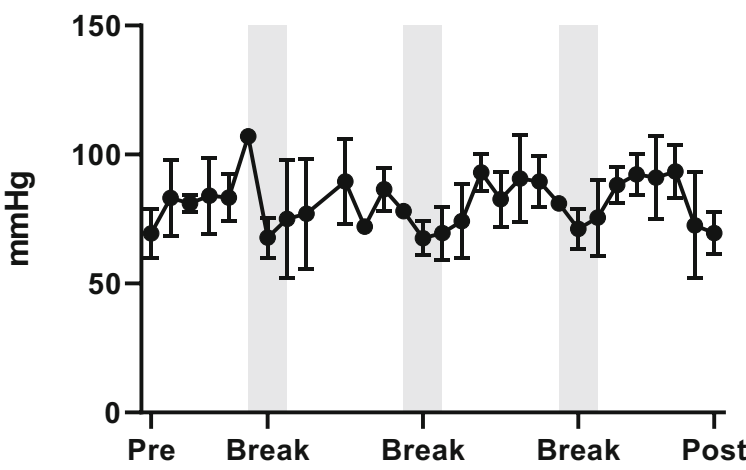




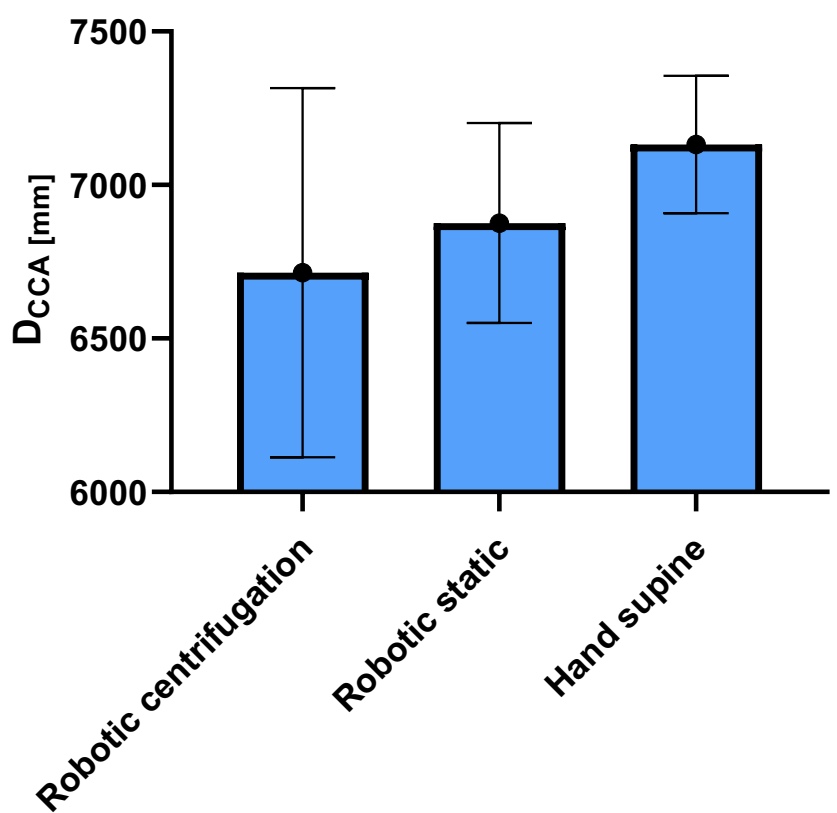

Condition

Fig. 8 Carotid diameter $\left(D_{C C A}\right)$ for all four conditions (hand supine, robotic static, hand vertical, robotic centrifugation)

variability for this study could be explained by a slightly difference in the measurement position (probably more proximal) and by the small sample size of the groups. The ultrasound measurement reproducibility is similar with an intrameasurement's variability of $0.2 \mathrm{~mm}$ and an intermeasurement variability equals $60 \mu \mathrm{m}$ in both studies. If

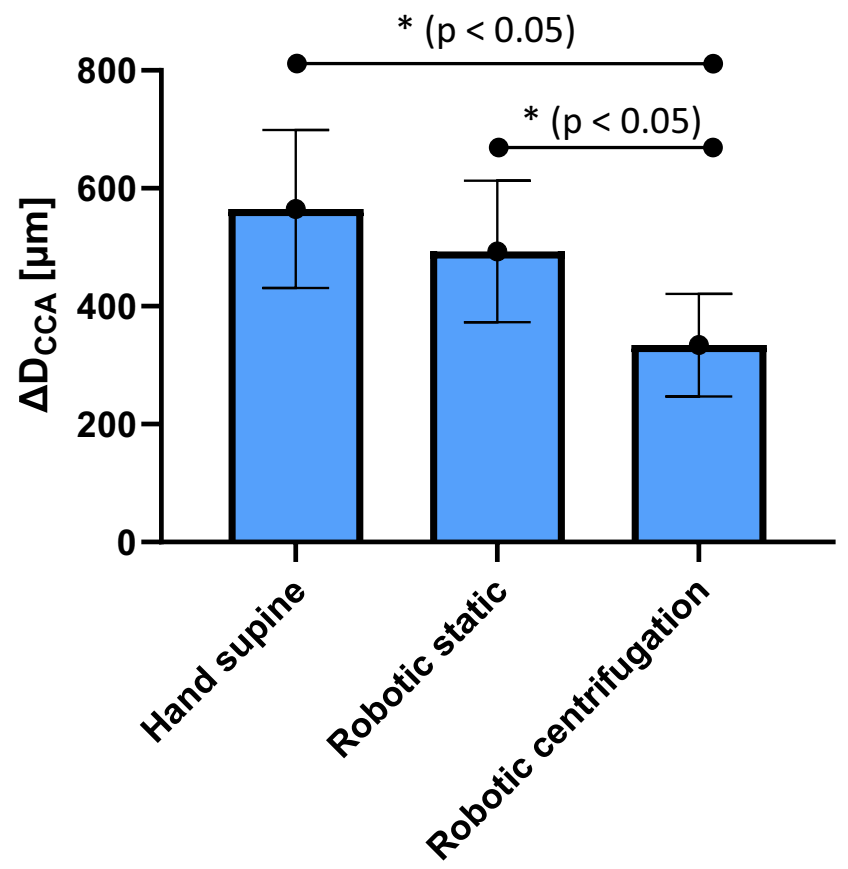

Condition

Fig. 9 Carotid distention $\left(\Delta D_{C C A}\right)$ for all four conditions (hand supine, robotic static, hand vertical, robotic centrifugation)

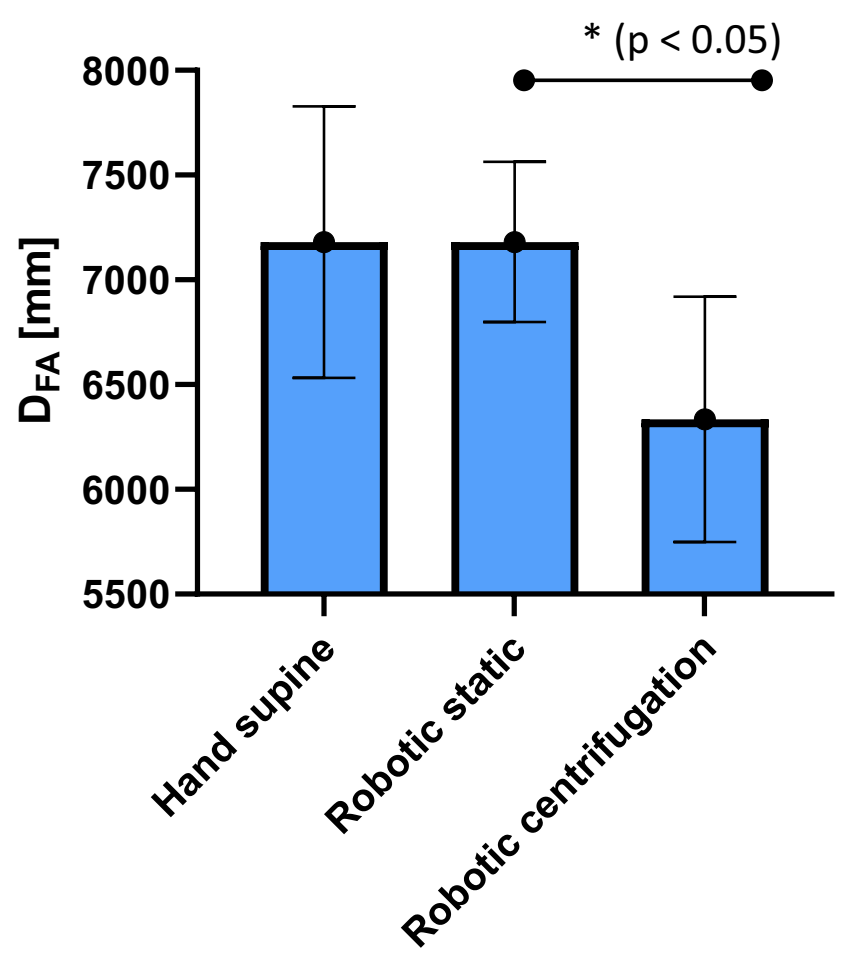

\section{Condition}

Fig. 10 Femoral diameter $\left(D_{F A}\right)$ for all four conditions (hand supine, robotic static, hand vertical, robotic centrifugation)

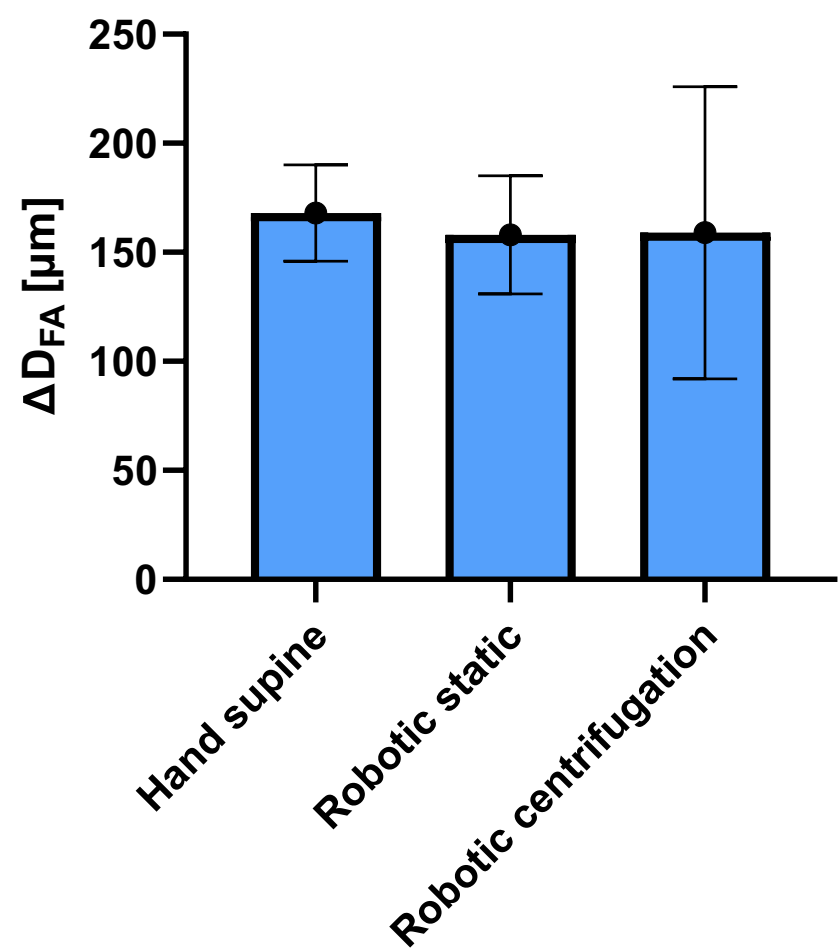

Condition

Fig. 11 Femoral distention $\left(\Delta D_{F A}\right)$ for all four conditions (hand supine, robotic static, hand vertical, robotic centrifugation) 


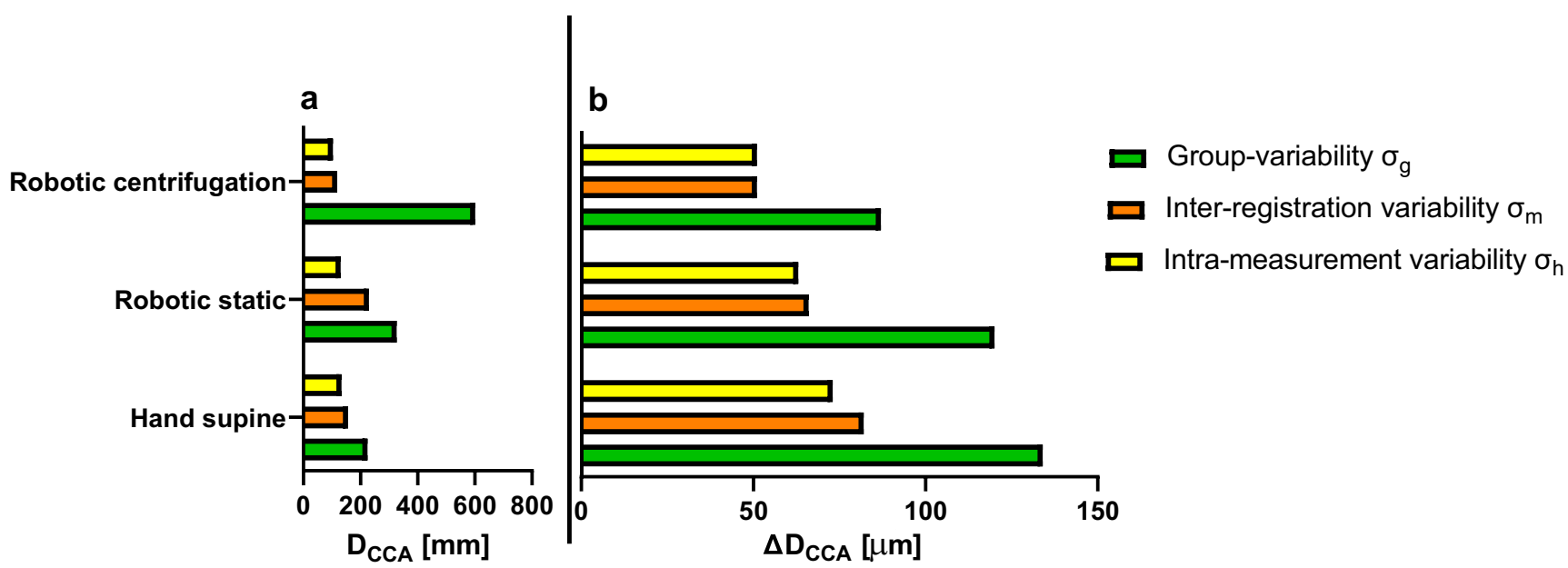

Fig. 12 Variability of $\mathbf{a}$ : carotid diameter measurements $\left(\boldsymbol{D}_{\boldsymbol{C C A}}\right)[\mathrm{mm}]$ and $\mathbf{b}$ : carotid distension $\left(\Delta \boldsymbol{D}_{\boldsymbol{C} C \boldsymbol{A}}\right)[\mu \mathrm{m}]$ for the three different measurement conditions

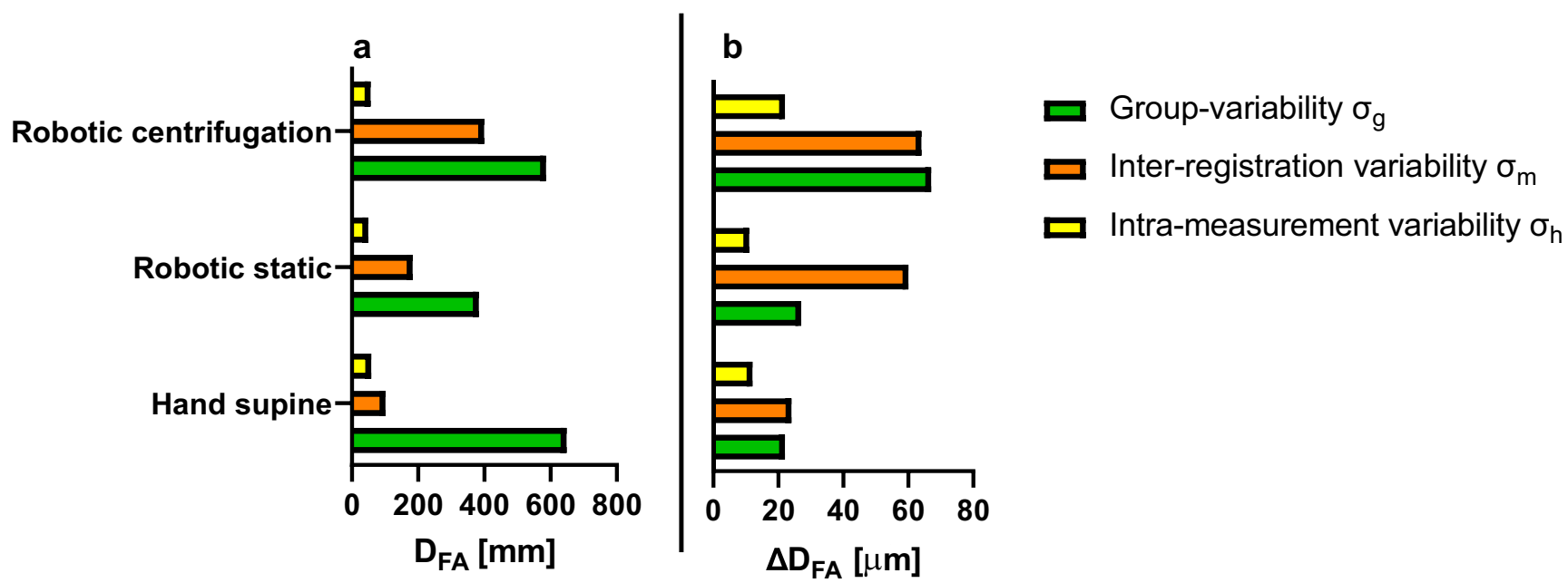

Fig. 13 Variability of a: femoral diameter measurements $\left(D_{F A}\right)[\mathrm{mm}]$ and $\mathbf{b}$ : femoral distension $\left(\Delta D_{F A}\right)[\mu \mathrm{m}]$ for the three different measurement conditions

converted to percentage of the diameter, the distension values for the femoral are also matching with $2.3 \pm 0.2 \%$ and $2.7 \pm$ $1.4 \%$ in this study, and, in Leguy et al., respectively.

A good reproducibility and precision of the remotely controlled vascular ultrasound assessment under centrifugation could be demonstrated. No significant differences between the measurements performed by hand in supine position and the ones obtained with the ultrasound probe attached at the robotic arm in supine position without centrifugation were found. These results demonstrate that a good positioning of the probe could be reached using the robotic arm, and, that the pressure applied to the subject skin by the probe (limited to 20 Newton) did not disturb the quality of the ultrasound measurements. Considering the inter-measurement variability $\sigma_{\mathrm{h}}$ as a metric for the precision of the measurements, our results show that the measurements performed with the robotic arm during
Table 1 Variability of carotid diameter measurements (in $\mu \mathrm{m}$ ) for the three different measurement conditions

\begin{tabular}{llll}
\hline & Hand supine & Robotic static & Robotic centrifugation \\
\hline Mean Diameter & 7132 & 6876 & 6714 \\
Group-variability, $\sigma_{g}$ & \pm 224 & \pm 326 & \pm 601 \\
Inter-registration variability, $\sigma_{m}$ & \pm 156 & \pm 229 & \pm 118 \\
Intra-measurement variability, $\sigma_{h}$ & \pm 133 & \pm 130 & \pm 103 \\
\hline
\end{tabular}


Table 2 Variability of femoral diameter measurements (in $\mu \mathrm{m}$ ) for the three different measurement conditions

\begin{tabular}{llll}
\hline & Hand supine & Robotic static & Robotic centrifugation \\
\hline Mean Diameter & 7180 & 7181 & 6334 \\
Group- variability, $\boldsymbol{\sigma}_{\boldsymbol{g}}$ & \pm 648 & \pm 383 & \pm 586 \\
Inter-registration variability, $\boldsymbol{\sigma}_{\boldsymbol{m}}$ & \pm 101 & \pm 183 & \pm 399 \\
Intra-measurement variability, $\boldsymbol{\sigma}_{\boldsymbol{h}}$ & \pm 58 & \pm 49 & \pm 56 \\
\hline
\end{tabular}

Table 3 Variability of carotid distention measurements (in $\mu \mathrm{m}$ ) for the three different measurement conditions

\begin{tabular}{llll}
\hline & Hand supine & Robotic static & Robotic centrifugation \\
\hline Mean Distension & 565 & 493 & 334 \\
Group- variability, $\boldsymbol{\sigma}_{\boldsymbol{g}}$ & \pm 134 & \pm 120 & \pm 87 \\
Inter-registration variability, $\boldsymbol{\sigma}_{\boldsymbol{m}}$ & \pm 82 & \pm 66 & \pm 51 \\
Intra-measurement variability, $\boldsymbol{\sigma}_{\boldsymbol{h}}$ & \pm 73 & \pm 63 & \pm 51 \\
\hline
\end{tabular}

centrifugation are as precise as the measurements obtained under non-moving conditions. Furthermore, a good reproducibility is reflected by the low inter-measurement variability $\sigma_{\mathrm{m}}$ between the repeated centrifugal runs. Overall, the expected relationship between the variability parameters, $\sigma_{\mathrm{h}}<\sigma_{\mathrm{m}}<\sigma_{\mathrm{g}}$, is met except for distension at the femoral artery (for which the capacity of functional ultrasound detection is nearly reached).

The vascular parameters could be quantitatively assessed using the robotic-arm in supine position with and without centrifugation, and, demonstrate physiological behaviour. The diameter of the carotid artery was maintained during centrifugation, that can be explained by the autoregulation of blood flow to the brain and the shear-stress regulated CCA diameter regulation. As expected, the distention of the carotid artery decreased during centrifugation following the course of the decreased pulse-pressure (the carotid artery being characterised by mainly linear-elastic walls). Furthermore, the obtained decrease in the femoral diameter during centrifugation might be explained by the vasoconstriction induced by blood pooling towards the lower extremity, as during standing $(19,20)$. Due to the limited sample size and short exposure to $\mathrm{g}$-load these findings require further evaluation in order to rich higher level of statistical significances.

From 9 subjects that we tested in this pilot trial, one subject showed pre-syncope symptoms during centrifugation. This pre-syncope may have been caused by a vasovagal reaction that could be triggered by some additional pressure applied on the baroreceptor of the carotid artery by the remotely controlled robotic system. Being aware of this potential risk, each subject had been tested for a potential drop of heart rate when pressure is applied by the ultrasound probe at the carotid artery (close to the carotid sinus) under supine position. No subjects presented with an increased heart-rate. However, these tests could not be performed during the centrifugation (for technical as well as safety reason) therefore such a risk for presyncope could not be avoided. Although limited in maximal applicable pressure, the visual control alone using cameras during centrifugation is not optimal. A special attention to the first minute of centrifugation while the robotic arm is used at the carotid level is being recommended.

Potential steps to optimize a robotic controlled ultrasound system are a) distance measurement between probe and participant's skin b) a 3-dimensional visualisation of the robotic arm (e.g. using virtual reality googles) to increase accuracy of probe positioning and c) a haptic feedback system to precisely control the applied pressure of the probe. Such technical developments would allow the imaging of internal organs (e.g. heart), which requires a dynamic and precise control of probe position and pressure during centrifugation.

We conclude that with the current state-of-the-art robotic systems ultrasound measurements during centrifugation are feasible by trained examiners for peripheral arteries as the
Table 4 Variability of femoral distention measurements (in $\mu \mathrm{m}$ ) for the three different measurement conditions

\begin{tabular}{llll}
\hline & Hand supine & Robotic static & Robotic centrifugation \\
\hline Mean Distension & 168 & 158 & 159 \\
Group-variability, $\boldsymbol{\sigma}_{\boldsymbol{g}}$ & \pm 22 & \pm 27 & \pm 67 \\
Inter-registration variability, $\boldsymbol{\sigma}_{\boldsymbol{m}}$ & \pm 24 & \pm 60 & \pm 64 \\
Intra-measurement variability, $\boldsymbol{\sigma}_{\boldsymbol{h}}$ & \pm 12 & \pm 11 & \pm 22 \\
\hline
\end{tabular}


carotid artery and femoral artery. Furthermore, the successful measurements during centrifugation demonstrated reproducibility and precision that are as good as under static conditions. A better measurement success ratio during centrifugation could be achieved by better manipulator feedback.

Acknowledgements Our sincere thanks go to all volunteers who participated in this study. Furthermore, we would like to thank Alexandra Noppe, Melanie v.d. Wiesche and the rest of the DLR study team for their support during the recruitment process.

Author Contributions C.L., T.F., G.P. performed experiments; C.L. and T.F. analyzed data; T.F. and C.L. conception and design of research; T.F., C.L. and M.A. edited and revised manuscript; all authors approved final version of manuscript.

Funding Open Access funding enabled and organized by Projekt DEAL. This study was financed by DLR internal funding. The work of Carole Leguy was performed with the support of the Marie Curie International Out-going fellowship of the European's 7th Framework Programme for Research under contract number MC - IOF- 297967.

Data Availability Correspondence and requests for materials should be addressed to timo.frett@dlr.de

The datasets generated during and/or analysed during the current study are available from the corresponding author.

\section{Compliance with Ethical Standards}

Conflict of Interest Authors report no conflict of interest.

Open Access This article is licensed under a Creative Commons Attribution 4.0 International License, which permits use, sharing, adaptation, distribution and reproduction in any medium or format, as long as you give appropriate credit to the original author(s) and the source, provide a link to the Creative Commons licence, and indicate if changes were made. The images or other third party material in this article are included in the article's Creative Commons licence, unless indicated otherwise in a credit line to the material. If material is not included in the article's Creative Commons licence and your intended use is not permitted by statutory regulation or exceeds the permitted use, you will need to obtain permission directly from the copyright holder. To view a copy of this licence, visit http://creativecommons.org/licenses/by/4.0/.

\section{References}

Brands, P.J., Hoeks, A.P.G., Willigers, J., Willekes, C., Reneman, R.S.: An integrated system for the non-invasive assessment of vessel wall and hemodynamic properties of large arteries by means of ultrasound. Eur J Ultrasound. 9(3), 257-266 (1999)

Clement, G.: International roadmap for artificial gravity research. NPJ Microgravity. 3, 29 (2017)

Clément, G., Pavy-Le Traon, A.: Centrifugation as a countermeasure during actual and simulated microgravity: a review. Eur. J. Appl. Physiol. 92(3), 235-248 (2004)
Dogangil, G., Davies, B.L., Rodriguez, F., Baena, Y.: A review of medical robotics for minimally invasive soft tissue surgery. Proc Inst Mech Eng H. 224(5), 653-679 (2010)

Evans, C.R., Medina, M.G., Dwyer, A.M.: Telemedicine and telerobotics: from science fiction to reality. Updat. Surg. 70(3), 357-362 (2018)

Frett, T., Mayrhofer, M., Schwandtner, J., Anken, R., Petrat, G.: An innovative short arm centrifuge for future studies on the effects of artificial gravity on the human body. Microgravity Sci Technol. 26(4), 249-255 (2014a)

Frett, T., et al.: DLR-AG Facilities \& Research Plans. (2014b)

Frett, T., Petrat, G., van Loon, J.J.W.A., Hemmersbach, R., Anken, R.: Hypergravity facilities in the ESA ground-based facility program current research activities and future tasks. Microgravity Sci Technol. 28(3), 205-214 (2016)

Goswami, N., Bruner, M., Xu, D., Bareille, M.P., Beck, A., HinghoferSzalkay, H., Blaber, A.P.: Short-arm human centrifugation with $0.4 \mathrm{~g}$ at eye and $0.75 \mathrm{~g}$ at heart level provides similar cerebrovascular and cardiovascular responses to standing. Eur. J. Appl. Physiol. 115(7), 1569-1575 (2015)

Gunga, H.-C., et al.: The cardiovascular system in space, in Cardiovascular System, Red Blood Cells, and Oxygen Transport in Microgravity. Springer. p. 11-34 (2016)

Iwase, S., et al.: Effect of centrifuge-induced artificial gravity and ergometric exercise on cardiovascular deconditioning, myatrophy, and bone metabolism by simulated microgravity. Proceedings of Annual Meeting of the Physiological Society of Japan, 2007: p. 189-189 (2007)

Lee, S.M.C., et al.: Orthostatic intolerance after ISS and space shuttle missions. Aerosp Med Hum Perform. 86(12), A54-A67 (2015)

Leguy, C.A., et al.: Model-based assessment of dynamic arterial blood volume flow from ultrasound measurements. Med. Biol. Eng. Comput. 47(6), 641-648 (2009)

Linnarsson, D., et al.: Effects of an artificial gravity countermeasure on orthostatic tolerance, blood volumes and aerobic power after shortterm bed rest (BR-AG1). J Appl Physiol (1985). 118(1), 29-35 (2015)

Marescaux, J., Rubino, F.: Telesurgery, telementoring, virtual surgery, and telerobotics. Curr Urol Rep. 4(2), 109-113 (2003)

Meinders, J.M., Hoeks, A.P.: Simultaneous assessment of diameter and pressure waveforms in the carotid artery. Ultrasound Med. Biol. 30(2), 147-154 (2004)

Simón, X., Engle, J., Clark, T.K.: Artificial Gravity System Configurations Informed by Physiological Spin-Tolerance Research, In: 2018 AIAA SPACE and Astronautics Forum and Exposition (n.d.)

Stenger, M.B., Evans, J.M., Knapp, C.F., Lee, S.M.C., Phillips, T.R., Perez, S.A., Moore Jr., A.D., Paloski, W.H., Platts, S.H.: Artificial gravity training reduces bed rest-induced cardiovascular deconditioning. Eur. J. Appl. Physiol. 112(2), 605-616 (2012)

Verma, A.K., Xu, D., Bruner, M., Garg, A., Goswami, N., Blaber, A.P., Tavakolian, K.: Comparison of autonomic control of blood pressure during standing and artificial gravity induced via short-arm human centrifuge. Front. Physiol. 9, 712 (2018)

Publisher's Note Springer Nature remains neutral with regard to jurisdictional claims in published maps and institutional affiliations. 